\title{
Pulmonary tuberculosis and COVID-19 coinfection: A new medical challenge
}

\author{
Miguel Osejo-Betancourt, Sebastian Molina-Paez, Mariana Rubio-Romero \\ Hospital Santa Clara, Bogotá, Colombia
}

\begin{abstract}
Radiological findings in chest radiography and respiratory symptomatology represent a great challenge of interpretation during the COVID-19 (Coronavirus Disease 2019) pandemic, as their patterns can generate uncertainty at the time of diagnosis. This case highlights the importance in achieving an adequate correlation between diagnostic imaging and the clinical picture. We present a male adult who was admitted for 8 days of respiratory symptoms. Management with steroids was initiated according to the RECOVERY (Randomized Evaluation of COVID-19 Therapy) protocol and later confirmation of SARS-CoV-2 infection was received. In the following weeks, he deteriorated slowly and progressively clinical-
\end{abstract}

\section{Correspondence: Miguel Osejo, Hospital Santa Clara, Avenida Carrera 14 \#34-17, Teusaquillo, Bogotá 111311, Colombia. Tel. +57.3044964089. \\ E-mail: mosejob@unbosque.edu.co \\ Key words: Tuberculosis; COVID-19; SARS-CoV-2; cavitation; pneu- monia. \\ Contributions: All the authors made a substantive intellectual contri- bution, performed part of the experiments. All the authors have read and approved the final version of the manuscript and agreed to be accountable for all aspects of the work. \\ Conflict of interest: The authors declare that they have no competing interests, and all authors confirm accuracy.}

Ethical approval: No Ethical Committee approval was required for this case report by the Department, because this article does not contain any studies with human participants or animals. Written informed consent was obtained from the patient for publication of this case report and accompanying images. A copy of the written consent is available for review on request.

Received for publication: 7 August 2021.

Accepted for publication: 8 October 2021.

Publisher's note: All claims expressed in this article are solely those of the authors and do not necessarily represent those of their affiliated organizations, or those of the publisher, the editors and the reviewers. Any product that may be evaluated in this article or claim that may be made by its manufacturer is not guaranteed or endorsed by the publisher.

${ }^{\circ}$ Copyright: the Author(s), 2021

Licensee PAGEPress, Italy

Monaldi Archives for Chest Disease 2022; 92:2046

doi: 10.4081/monaldi.2021.2046

This article is distributed under the terms of the Creative Commons Attribution-NonCommercial International License (CC BY-NC 4.0) which permits any noncommercial use, distribution, and reproduction in any medium, provided the original author(s) and source are credited. ly, without reaching respiratory failure. Imaging showed a thickwalled cavitation in the right lower lobe. Tuberculosis was suspected and confirmed. The uniqueness of this case of COVID-19 coinfection in a patient with undiagnosed tuberculosis, represents a diagnostic and clinical management challenge, where the proper interpretation of chest radiology is a fundamental tool.

\section{Introduction}

In 2019, the first cases of pneumonia attributed to a new virus, known as SARS-CoV- 2 (severe acute respiratory syndrome coronavirus 2), were reported in Wuhan [1]. Infection by this virus, identified as a coronavirus, is characterized by a high rate of transmissibility and a wide spectrum of manifestations, from mild symptoms to life-threatening disease [1,2]. Tuberculosis (TB) is an infectious disease caused by the bacillus Mycobacterium tuberculosis, which has similarities with SARS-CoV-2 in terms of its transmission mechanism and symptomatology. According to global statistics for 2019, there were an estimated 10 million new TB cases and 1.2 million TB deaths in non-HIV patients [3].

In the context of the SARS-CoV-2 pandemic with current rising incidence, and high prevalence of TB in Latin America, co-infection should be considered. This is important because there is the possibility of co-infection by tuberculosis and SARS-CoV-2, causing a more severe presentation of COVID-19 (Coronavirus Disease 2019), which could be attributed to the different mechanisms of parenchymal damage of both etiologies and the generated immune response [4]. It is essential to understand how these respiratory infections are related and the impact that these two etiologies has on the evolution and prognosis of patients [5].

Due to the insidious clinical manifestations of tuberculosis and the high incidence of COVID-19, tuberculosis is currently the first disease to be ruled out in new or exacerbated respiratory symptoms; the diagnosis of tuberculosis is delayed and is only suspected if there is no clinical improvement or if there is a suggestive radiological alteration [5]. This is reflected in the increase of mortality due to tuberculosis between 0.2-0.4 million globally during 2020 [3]. In this context, the aim of this case report is to describe the clinical presentation of a patient co-infected with tuberculosis and COVID19 , highlighting the variability of the course of the disease, the management and diagnostic challenge that this represents.

\section{Case Report}

A 71-year-old male patient, former smoker of tobacco and bazuco (low-cost paste based on cocaine sulfate and other components such as kerosene, ether and silicates), who consulted the Santa Clara Hospital in the city of Bogota, Colombia, for 8 days 
of general malaise, anosmia, dysgeusia, fever, dry cough, later with dyspnea and cyanosis. On admission, oxygen saturation was $80 \%$, so supplemental oxygen was started with a non-rebreathing mask. Given the actual context, it was considered suspicious for SARS$\mathrm{CoV}-2$ infection, reverse transcription-polymerase chain reaction (RT-PCR) was requested and dexamethasone was started according to the RECOVERY (Randomized Evaluation of COVID-19 Therapy) protocol, as well as clarithromycin and ampicillin sulbactam, due to the suspicion of bacterial coinfection.

The admission laboratory tests reported a complete blood count without leukocytosis, with elevated troponins and D-dimer, chest X-ray with alveolar opacities in both lung fields and positive RT-PCR result for SARS-CoV-2. During hospitalization he maintained a stable clinical condition, without respiratory failure, but he did not tolerate the withdrawal of supplemental oxygen. The patient was initially considered to have lung damage associated with the use of inhaled drugs, with suspicion of tobacco-related interstitial lung disease; however, in the context of the pandemic and the clinical condition of the patient, pulmonary function tests were contraindicated.

Three weeks after, he presented worsening of dyspnea and persistent fever, so a control chest X-ray was taken, which showed no increase in opacities but with an image of irregular borders at the level of the right pulmonary hilum, high resolution computed tomography (HRCT) was requested, which showed bilateral apical consolidations with some centrolobular nodules, right basal consolidation (Figure 1), and a thick-walled cavitation in the upper segment of the right lower lobe (Figures 2 and 3). Healthcare-associated pneumonia with a necrotizing component was initially considered, so piperacillin tazobactam was started and sputum smear microscopy and Xpert-MTB/RIF ${ }^{\circledR}$ and fungal studies were requested. The sputum smear microscopy was positive for acidfast bacilli (AFB) and Xpert-MTB/RIF ${ }^{\circledR}$ with Mycobacterium tuberculosis medium detected without resistance to rifampicin, confirming the diagnosis of pulmonary tuberculosis, which could be tuberculous pneumonia and treatment was started with rifampicin, isoniazid, pyrazinamide and ethambutol. In addition, an HIV (human immunodeficiency virus) test was performed with a non-reactive result.

With the beginning of the treatment, he presented improvement of dyspnea and he was able to change from a non-rebreathing mask to a low-flow nasal cannula. After 3 weeks of treatment, the patient was in good general condition, with normal control laboratory tests, control AFB smears with negative results and tolerating the oral route. Finally, it was decided to discharge the patient with a plan to continue outpatient treatment and follow-up appointments with infectiology and pulmonology.

\section{Discussion}

The SARS-CoV-2 pandemic has generated an unprecedented state of global health emergency, which has had a great impact particularly in areas with high incidence and prevalence rates of tuberculosis, such as Southeast Asia, Africa, and the Americas [3,6]. Despite the decline in tuberculosis incidence and mortality figures that had been occurring in recent years, the statistics rose again during the SARS-CoV-2 pandemic [5]. It has been observed that the SARS-CoV-2 has had a socio-economic impact on the burden of TB disease, as it has substantially reduced the screening, identification and follow-up of TB patients. This is because security measures for COVID-19 have made access to health services more difficult, and

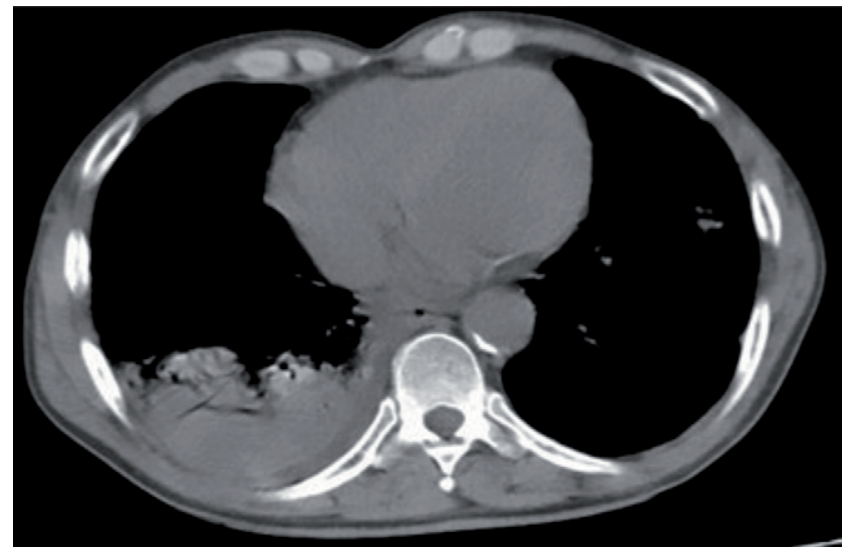

Figure 1. HRCT showing consolidation in the right lower lobe, with pleural effusion and passive atelectasis.

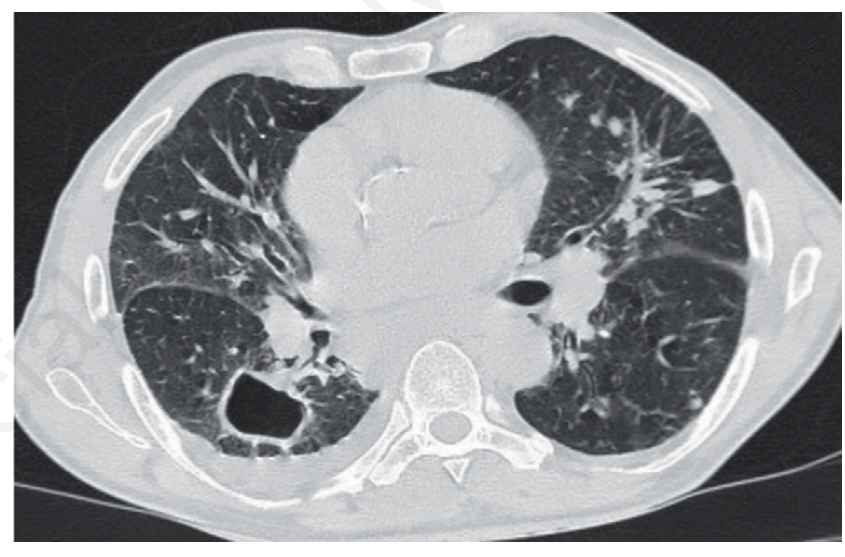

Figure 2. HRCT axial section showing thick-walled cavitation in the upper segment of the right lower lobe.

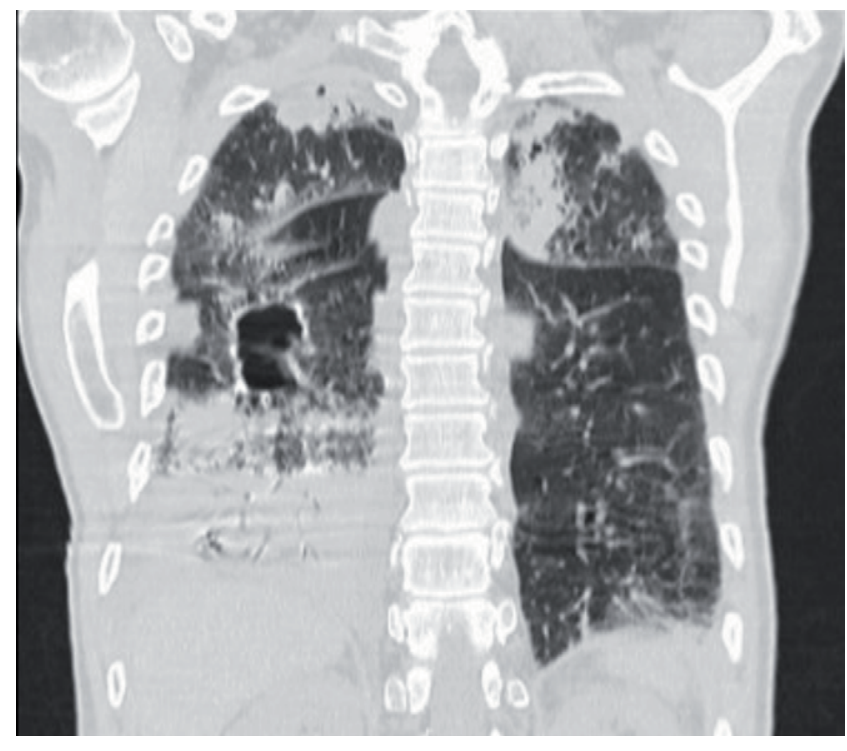

Figure 3. HRCT coronal slice showing thick-walled cavitation in the superior segment of the right lower lobe. 
resources for the care of these patients has been reduced by the increased demand for care in COVID-19 patients [7].

Tuberculosis is an infectious disease caused by the bacillus Mycobacterium tuberculosis. Its average size is 1-4 micrometers and its transmission mechanism is through aerosols produced mainly by individuals with a high bacilliferous load. The most effective mechanism of production of aerosols is through coughing, with active pulmonary and laryngeal infection producing the most aerosols $[3,4]$. This has the capacity to develop a chronic disease of the pulmonary parenchyma, with discrete clinic, but with great pathogenic potential [5]. SARS-CoV-2 is a virus of the Coronaviridae family, with a size of 6-12 micrometers in diameter, single-stranded RNA (ribonucleic acid) and a viral genome of 8.4$12 \mathrm{kDa}$ in size, considered the largest [1,2]. Its main transmission mechanism is by inhalation of aerosols, other mechanisms are contact with fomites and direct contact with mucous membranes [1,2]. Given the similarities between microorganisms a co-infection should be considered in the initial presentation of both entities in areas of high prevalence.

The clinical course of these infections are highly variable and the key to understanding the interactions of these etiologies within the co-infected host lies in the pathophysiology and immune response $[8,9]$. TB is characterized by a cellular response mediated mainly by alveolar macrophages that phagocytose the bacillus, which alters the function of the phagosome, preventing its destruction and allowing its replication [8,9]. If the immune system does not control the infection, it moves to the pulmonary parenchyma where it generates a multicellular response that will end in the formation of a granuloma [8,9]. This manifestation does not stop replication, but encapsulates the mycobacteria preventing the spread of the disease [8].

Knowledge about the pathophysiology and immune response of SARS-CoV-2 infection is still scarce, however some observational studies have established that the response varies according to the phase and severity of the disease. Initially, an INF-1 (type 1 interferon) humoral response is generated, elevating different cytokines (IL6, IL-8, INF-y, etc.), which recruit macrophages, neutrophils, T-CD4 lymphocytes and natural killer, which will be reflected by leukocytosis with neutrophilia and the elevation of markers such as ferritin and C-reactive protein, which have been validated as prognostic markers [10]. In severe disease, a deregulated response is evidenced in which the INF-1 and INF-2 responses do not reach a balance, which can cause increased tissue damage, saturate the cellular response mechanisms, evidencing a decrease in the cell count of the lymphoid line, including macrophages, dendritic cells and highlighting lymphopenia, which could induce the activation of latent tuberculosis infection (LTBI) [11,12].

According to studies, coinfection with TB is associated with a more severe presentation of COVID-19, with greater complications [13]. This is related to the residual pulmonary abnormalities produced by $\mathrm{TB}$, which together with the compromise of the immune response favors other respiratory infections [11,13]. LTBI is characterized by maintaining a persistent activation of the humoral and cellular immune response, in which cytokines and CD4 $\mathrm{T}$ lymphocytes stand out, which also mediate the immune response against SARS-CoV-2 [11].

Radiological findings are a fundamental part of the diagnosis in both entities. In primary tuberculosis the most common finding is mediastinal and hilar lymphadenopathy, as well as slowly resolving parenchymal consolidations and pleural effusion. In tuberculosis reactivation, thick-walled irregular cavitations and consolidations predominate, and miliary tuberculosis is characterized by diffuse 1-3 mm nodules [14]. Regarding COVID-19, vari- ous radiological findings have been described. However, the typical abnormalities are bilateral and peripheral multifocal groundglass opacities, crazy paving pattern, progressive consolidations and organizing pneumonia. It is important to mention that these radiological findings on HRCT are progressive and reflect the inflammation caused by SARS-CoV-2 [15]. Both entities have a wide spectrum of radiological manifestations that may overlap in the patient, making it unlikely that co-infection is investigated, delaying the diagnosis as it happened with the patient.

In a recently published paper, a cohort of 49 patients from 8 countries with TB/COVID- 19 was described, the time of diagnosis was highly variable, but the follow-up with images helped them to think about the diagnosis of tuberculosis and $47.9 \%$ presented cavitation, like our patient [16].

Currently, the best evidence of decreased mortality in patients with COVID-19 requiring hospitalization and supplemental oxygen or in patients on mechanical ventilation is the RECOVERY protocol. This consists of the use of intravenous dexamethasone $6 \mathrm{mg}$ daily for 7 to 10 days after day 7 of illness [17], which was performed in our patient. Additionally, it has been observed that the use of corticosteroids at doses higher than $15 \mathrm{mg} / \mathrm{kg}$ for 2 to 4 weeks increases the risk of reactivation of LTBI up to 4.9 times; therefore, the administration of corticosteroids in the context of COVID-19 could weaken host immunity [18]. This could lead to reactivation of LTBI or progression of active pulmonary TB undiagnosed at the time of admission. Accordingly, TB patients are less likely to generate an adequate response against SARS-CoV-2, and SARS-CoV-2 infection generates a suitable environment for TB reactivation or progression by altering the cellular and humoral response [18]. In 2 European cohorts that included 69 patients, a preliminary analysis was made of 8 deaths in TB/COVID-19 patients, in all cases the clinical condition and prognosis worsened, it is remarkable that the deaths were more common in older patients, males and with comorbidities, which is already expected from COVID-19, but it seems that TB is not a determining factor in mortality. The analysis included immigrants from low- income countries, that were younger, but with other risk factors such as smoking, alcoholism and HIV infection; which favors the appearance of infection with MDR/XDR-TB, which require a longer treatment time, other drugs, which increase health costs, and in the long term may increase morbidity and mortality in this group of patients [19]. Although studies and case reports of this coinfection are increasing, further studies are still required to adequately understand how the two diseases may synergize in morbidity and mortality.

\section{Conclusions}

The management of TB/COVID-19 patients is a topic of utmost importance in countries with a high prevalence of these diseases, as is the case in most Latin American countries. Because the immune system becomes unable to generate an adequate response to this coinfection, an imbalance in the immune response occurs, progressing latent tuberculosis to active infection and increasing the damage caused by SARS-CoV-2. Overlapping radiological manifestations cause confusion and delay diagnosis, increasing hospital length of stay, costs and patient morbidity and mortality. In regions with a high prevalence of $\mathrm{TB}$, it may be suggested to perform screening tests for tuberculosis in hospitalized patients, if after 1 or 2 weeks of conventional COVID-19 treatment they do not present an adequate clinical evolution. Likewise, screening should be considered in patients with pneumonia or respiratory distress. 


\section{References}

1. Shereen MA, Khan S, Kazmi A, et al. COVID-19 infection: Emergence, transmission, and characteristics of human coronaviruses. J Adv Res 2020;24:91-98.

2. Umakanthan S, Sahu P, Ranade AV, et al. Origin, transmission, diagnosis and management of coronavirus disease 2019 (COVID-19). Postgrad M J 2020;96:753.

3. World Health Organization. Global tuberculosis report 2020. Last access 15 December 2020 Available from: https://www.who.int/teams/global-tuberculosisprogramme/tb-reports/global-tuberculosis-report-2020

4. Wei M, Zhao Y, QianZ, et al. Pneumonia caused by mycobacterium tuberculosis. Microbes infect 2020;22:278-84.

5. Ufimtseva E, Eremeeva N, Vakhrusheva D, Skornyakov S. Mycobacterium tuberculosis shape and size variations in alveolar macrophages of tuberculosis patients. Eur Respir J 2019;54:PA4605.

6. Churchyard G, Kim P, Shah NS, et al. What we know about tuberculosis transmission: An overview. J Infect Dis 2017;216:S629-35.

7. Migliori GB, Thong PM, Alffenaar J-W, et al. Gauging the impact of the COVID-19 pandemic on tuberculosis services: a global study. Eur Respir J 2021;58:2101786.

8. Vanzetti CP, Salvo CP, Kuschner P, Brusca S. [Coinfección tuberculosis y COVID-19].[Article in Spanish]. Medicina (B. Aires) 2020;80:s100-3.

9. Pai M, Behr MA, Dowdy D, et al. Tuberculosis. Nat Rev Dis Primers 2016;2:16076.
10. Osuchowski MF, Winkler MS, Skirecki T, et al. The COVID19 puzzle: deciphering pathophysiology and phenotypes of a new disease entity. Lancet Respir Med 2021;9:622-42.

11. Mousquer GT, Peres A, Fiegenbaum M. Pathology of TB/COVID-19 Co-infection: The phantom menace. Tuberculosis 2021;126:102020.

12. Petrone L, Petruccioli E, Vanini V, et al. Coinfection of tuberculosis and COVID-19 limits the ability to in vitro respond to SARS-CoV-2. Int J Infect Dis 2021;113:S82-7.

13. Udwadia ZF, Vora A, Tripathi AR, et al. COVID-19Tuberculosis interactions: When dark forces collide. Indian J Tuberc 2020;67:S155-62.

14. Nachiappan AC, Rahbar K, Shi X, et al. Pulmonary tuberculosis: Role of radiology in diagnosis and management. Radiographics 2017;37:52-72.

15. Kwee TC, Kwee RM. Chest CT in Covid-19: What the radiologist needs to know. Radiographics 2020;40:1848-65.

16. Tadolini M, Codecasa LR, García-García JM, et al. Active tuberculosis, sequelae and COVID-19 co-infection: First cohort of 49 cases. Eur Respir J 2020;56:2001398.

17. RECOVERY Collaborative Group, Horby P, Lim WS, et al. Dexamethasone in Hospitalized Patients with Covid-19. N Engl J Med 2021;384:693-704.

18. Gopalaswamy R, Subbian S. Corticosteroids for covid-19 therapy: Potential implications on tuberculosis. Int $\mathrm{J}$ Mol Sci 2021;22:3773.

19. Motta I, Centis R, D’Ambrosio L, et al. Tuberculosis, COVID19 and migrants: Preliminary analysis of deaths occurring in 69 patients from two cohorts. Pulmonology 2020;26:233-40. 